\title{
Silencing of Entamoeba histolytica Glucosamine 6-Phosphate Isomerase by RNA Interference Inhibits the Formation of Cyst-Like Structures
}

\author{
Hugo Aguilar-Díaz, Juan Pedro Laclette, and Julio César Carrero \\ Departamento de Inmunología, Instituto de Investigaciones Biomédicas, Universidad Nacional Autónoma de México, \\ A.P. 70228, Mexico City, DF, Mexico \\ Correspondence should be addressed to Julio César Carrero; carrero@servidor.unam.mx
}

Received 10 August 2012; Revised 11 November 2012; Accepted 20 November 2012

Academic Editor: Luis I. Terrazas

Copyright (C) 2013 Hugo Aguilar-Díaz et al. This is an open access article distributed under the Creative Commons Attribution License, which permits unrestricted use, distribution, and reproduction in any medium, provided the original work is properly cited.

\begin{abstract}
Encystment is an essential process in the biological cycle of the human parasite Entamoeba histolytica. In the present study, we evaluated the participation of E. histolytica Gln6Pi in the formation of amoeba cyst-like structures by RNA interference assay. Amoeba trophozoites transfected with two Gln6Pi siRNAs reduced the expression of the enzyme in 85\%, which was confirmed by western blot using an anti-Gln6Pi antibody. The E. histolytica Gln6Pi knockdown with the mix of both siRNAs resulted in the loss of its capacity to form cyst-like structures (CLSs) and develop a chitin wall under hydrogen peroxide treatment, as evidenced by absence of both resistance to detergent treatment and calcofluor staining. Thus, only $5 \%$ of treated trophozoites were converted to CLS, from which only $15 \%$ were calcofluor stained. These results represent an advance in the understanding of chitin biosynthesis in E. histolytica and provide insight into the encystment process in this parasite, which could allow for the developing of new control strategies for this parasite.
\end{abstract}

\section{Introduction}

The intestinal protozoan parasite Entamoeba histolytica, the causal agent of human amoebiasis around the word, is considered a serious problem of public health mainly in developing countries. Amoebiasis is a major source of morbidity and mortality with estimate of 50 million people infected annually [1]. The E. histolytica life cycle includes two biological forms: the trophozoite and the cyst. The cyst is the infective form and has a tetranucleated structure surrounded by a chitin wall highly resistant to adverse environment conditions [2]. In spite of numerous in vitro studies focused on obtaining the infective mature forms of E. histolytica cysts, no reproducible procedure has yet been developed. However, a recent study by our group achieved induction of cyst-like structures (CLS) with a characteristic chitin thick wall, refringency, multinucleation (even a few of CLS with 4 nuclei) and chromatoid bodies by treatment of trophozoites with hydrogen peroxide in the presence of trace amounts of several metallic dications
[3]. The CLS obtained was resistant to lysis by detergents and exposition to environmental conditions for several weeks (unpublished data). These results suggest that encystment pathways can be activated in E. histolytica trophozoites when they are exposed to oxidative stress, mainly hydrogen peroxide, as it has been suggested for differentiation in other cells (reviewed in [4]). In this regard, exposure to luminal conditions, such as reactive oxygen species from immune cells or intestinal microbiota, has been suggested to be involved in amoeba encystment $[5,6]$. In addition, a role for certain divalent cations as cofactors of enzymes involved in excystment and encystment has also been described [7]. However, the cellular events behind the synthesis of the chitin wall and, in general, the developmental conversion of $E$. histolytica, are still unknown.

The in vitro encystment of other protozoans such as Giardia lamblia and E. invadens is extensively studied (reviewed in [8]). During the encystment of G. lamblia, it has been 
observed that the filamentous structure of the cyst wall consists of a polymer of $\mathrm{N}$-acetylgalactosamine [9]. This polymer is synthesized from endogenous glucose by a biosynthetic pathway where the rate-limiting enzyme is glucosamine-6phosphate isomerase ( $\mathrm{Gln} 6 \mathrm{Pi}$ ), an enzyme that reversibly isomerizes fructose-6-phosphate to glucosamine-6-phosphate [10]. Accordingly, we also reported that in vitro induction of E. histolytica CLS is coupled to overexpression of a Gln6Pi, as determined by RT-PCR [3]. However, solid evidence of the functional participation of this enzyme in amoeba encystment was not provided.

RNA interference (RNAi) method is a powerful tool for assessing and manipulating gene function. In this process, a double-stranded RNA (dsRNA) can initiate posttranscriptional sequence-specific silencing of cellular genes by mRNA degradation [11]. The mechanism of RNAi may have evolved as a defense against viruses and transposable elements with dsRNA intermediates $[12,13]$. In this process, the small RNA intermediates, short interfering RNAs (siRNAs), result from dsRNA being cleaved at 21 to 23 nucleotide intervals [14] by RNase III-type [15] and are then incorporated into the RNA-induced silencing complex (RISC) and guided to their target mRNA, which is then cleaved by Argonaute proteins $[14,16]$. So far we know that the molecular RNAi machinery and siRNAs are present in E. histolytica and the silencing of genes in this parasite is relatively common (reviewed in $[17,18])$.

In this work, we evaluated the participation of Gln6Pi in the formation of the CLS by RNA interference assay. Our results show that under encystment induction treatment, the Gln6Pi knockdown in trophozoites results in the loss of the capacity of trophozoites to synthesize the chitin wall, and therefore, to transform into a CLS, suggesting a pivotal role for Gln6Pi in E. histolytica encystment.

\section{Material and Methods}

2.1. Parasite Cultures. E. histolytica trophozoites, HM-1: IMSS strain, were grown at $37^{\circ} \mathrm{C}$ in sterile TYI-S-33 medium supplemented with $10 \%$ adult bovine serum, $100 \mathrm{U} / \mathrm{mL}$ of penicillin, $100 \mathrm{mg} / \mathrm{mL}$ of streptomycin sulfate, and $3 \%$ of Diamond Vitamins [19].

2.2. Induction of Cyst-Like Structures. The induction of cystlike structures (CLS) was performed as previously reported [3]. In brief, trophozoites were chilled on ice for $5 \mathrm{~min}$ and harvested by centrifugation at $150 \times \mathrm{g}$ for $7 \mathrm{~min}$ at $4^{\circ} \mathrm{C}$. The cells obtained $\left(1 \times 10^{5}\right)$ were resuspended in $50 \mathrm{~mL}$ of fresh TYI-S-33 media in culture flasks and incubated at $37^{\circ} \mathrm{C}$. After $72 \mathrm{~h}$, trophozoites in log phase (approximately $5 \times$ $10^{6}$ cells) were treated with $4 \mathrm{mM}$ of a $30 \% \mathrm{H}_{2} \mathrm{O}_{2}$ solution containing different traces of several dications (cadmium $0.02 \mathrm{ppm}$, cobalt $0.02 \mathrm{ppm}$, copper $0.02 \mathrm{ppm}$, iron $0.1 \mathrm{ppm}$, nickel $0.02 \mathrm{ppm}$, lead $0.02 \mathrm{ppm}$, zinc $0.02 \mathrm{ppm}$ ) and other components (free sulfuric acid $40 \mathrm{ppm}$, chlorine $0.5 \mathrm{ppm}$, phosphate $5 \mathrm{ppm}$, and sulfate $2 \mathrm{ppm}$ ) (Merck UN 2014, Darmstadt, Germany). The cultures were then incubated at $37^{\circ} \mathrm{C}$ for $6 \mathrm{~h}$. After treatment, parasites were washed two times with phosphate buffered saline, counted under microscope and resuspended in PBS containing 0.5\% sarkosyl, and allowed to sit for $10 \mathrm{~min}$ at room temperature. After three washes as above, the detergent-resistant trophozoites were resuspended in PBS and counted again in a microscope. The conversion rate index was estimated as the percentage of parasites that were resistant to sarkosyl and therefore were converted from trophozoites to CLS. Three independent experiments by triplicate were carried out for each analysis.

2.3. Small Interference RNA (siRNA). Design of siRNAs was done by the Ambion Company (TX, USA) using a patented siRNA design algorithm applied to the E. histolytica $\mathrm{Gln} 6 \mathrm{Pi}$ gene sequence. Design was followed by an in silico analysis using the E. histolytica genome database in order to avoid matching of the candidate siRNAs with other expressed trophozoite genes. Two 21-nt siRNAs, Si 154-Gln6Pi (5'GGACAUGCAGUAUUAGGAUTT-3') and Si 229-Gln6Pi (5'GCUGGAGAAGUUUCAUUUATT-3'), were designed and finally purchased from Applied Biosystem (USA). A siRNA with a scrambled sequence, unable to induce degradation in any cellular mRNA, was used as control of specificity (siRNA-A:sc-37007, Santa Cruz Biotechnology, CA, USA).

2.4. RNA Interference Assay. The siRNAs (154-Gln6Pi and 229-Gln6Pi) were used individually and combined at amounts of $5,10,20$, and $40 \mu \mathrm{g}$ per culture. The siRNA-A with the scrambled sequence was used at $40 \mu \mathrm{g}$ per culture following the manufacturer's instructions. Transfection of the trophozoites was performed by soaking as previously reported [17]. In brief, $1 \times 10^{5}$ trophozoites were grown in $6 \mathrm{~mL}$ TYI-S33 media in culture flasks. Once reached $50 \%$ of confluence (about $5 \times 10^{5}$ cells), cultures were added with the siRNA, individually or mixed $(5,10,20$, and $40 \mu \mathrm{g}$ of each one), and incubated at $37^{\circ} \mathrm{C}$ for $16 \mathrm{~h}$. After the incubation, transfected trophozoites were induced to encyst by exposure to the treatment solution ( $4 \mathrm{mM} \mathrm{H}_{2} \mathrm{O}_{2}+$ cations, see above) during $6 \mathrm{~h}$. The cells were then extensively washed with PBS $\mathrm{pH} 7.4$ and the conversion rate index (percentage of CLS formed) was determined with $0.5 \%$ sarkosyl as described. Morphological evaluation and determination of chitin were carried out by observation under light microscope and calcofluor white staining, respectively.

2.5. Calcofluor White Staining. After the transfection with siRNA and CLS induction, calcofluor white M2R staining was performed (Sigma-Aldrich, USA). The treated cells resistant to detergents (CLS) were washed three times with PBS. Subsequent to centrifugation, pellet samples were placed onto microscope slides and several drops of $0.05 \%$ calcofluor white $\mathrm{M} 2 \mathrm{R}$ in distilled water were added. The sample was incubated for $10 \mathrm{~min}$ and the slide was observed under UV light using a fluorescence microscope.

2.6. Viability Assay. Viability was analyzed by determining the ability of CLS to convert fluorescein diacetate (FDA) to fluorescein. The method was previously used in the infectivity 
TABLE 1: Oligonucleotides and siRNAs used in this work for RT-PCR and interference of Gln6Pi in E. histolytica, respectively.

\begin{tabular}{|c|c|c|}
\hline Target gene & Primer name & Sequence $\left(5^{\prime}\right.$ to $\left.3^{\prime}\right)$ \\
\hline$g \ln 6 P i$ & Gln6PiEh-F & ATGTCATCCACAAACGAAAATATTC \\
\hline gnl6Pi & Gln6PiEh-R & CAATAGACATGGATTTATCATATC \\
\hline EhARF & EhARF-F & GTAGGACTTGATGCTGCC \\
\hline$E h A R F$ & EhARF-R & TCACCATTAGTTGCAC \\
\hline$g \ln 6 P i-m R N A$ & siRNA 154-Gln6Pi & GGACAUGCAGUAUUAGGAUTT \\
\hline$g \ln 6 P i-m R N A$ & Si 229-Gln6Pi & GCUGGAGAAGUUUCAUUUATT \\
\hline- & siRNA-A:sc37007 & - \\
\hline
\end{tabular}

Controls for constitutive expression (ARF primers) and specificity of interference (siRNA-A) are also shown.

determination of $G$. lamblia cysts [20]. The viability assays were performed in the cells after the transfection with siRNAs to discard the possibility of toxic effects, as well as after the treatment with $4 \mathrm{mM} \mathrm{H} \mathrm{H}_{2} \mathrm{O}_{2}$ and $0.5 \%$ sarkosyl. In brief, treated trophozoites were centrifuged, pelleted, and washed three times with PBS $\mathrm{pH}$ 7.4. Afterwards, the cells were counted and adjusted to $1 \times 10^{6} \mathrm{~mL}$ with PBS. Samples of $100 \mu \mathrm{L}$ were treated with $1.6 \mu \mathrm{L}$ of FDA stock solution $(2.5 \mu \mathrm{g} / \mu \mathrm{L}$ in acetone; Invitrogen, USA $)$, at room temperature for $8 \mathrm{~min}$. Percentage of viable cells was determined by counting the number of fluorescent cells under a fluorescence microscope with a BP350-460 filter.

2.7. RNA Extraction and RT-PCR. Total RNA was isolated from siRNA-transfected E. histolytica trophozoites with TRIZOL (Invitrogen, USA). The level of expression of the Gln6Pi gene was determined by RT-PCR using Super Script III One-Step kit (Invitrogen, USA) following the manufacturer's instructions. An amount of $900 \mathrm{ng}$ of total RNA was used for cDNA production and the PCR performed with primers previously designed to target the E. histolytica Gln6Pi sequence (TIGR Accession number XM_648225) [3] (Table 1). Amplification of E. histolytica mRNA ADP-ribosylation factor was used as control of constitutive expression (ARF; accession number XM_648949) [6, 21] (Table 1). The RTPCR products were run in $1.5 \%$ agarose gels and quantified by densitometry using the Bio-Imaging System MiniBis (BioRad, USA) and the Image J program (Image Processing and Analysis in Java) after ethidium bromide staining.

2.8. Western Blot. The levels of Gln6Pi protein expression in extracts from trophozoites transfected with the siRNAs were determined by western blotting. A total of $15 \mu \mathrm{g}$ protein extracts prepared with protease inhibitors were run in $10 \%$ SDS-polyacrylamide gels and transferred to nitrocellulose membranes. Protein extracts from untreated trophozoites and trophozoites transfected with siRNA-A (scrambled sequence) were used as controls of basal expression. The membranes were blocked with 0.3\% PBS-Tween-5\% BSA solution overnight and probed with a mouse anti-human Gln6Pi polyclonal antibody (GNPDA1, Affinity Bioreagents, USA) diluted $1: 500$ overnight at $4^{\circ} \mathrm{C}$. After washing with PBS-Tween, HRP-conjugated anti-mouse IgG A M (Zymed, USA) diluted 1:500 was incubated with the membranes during $2 \mathrm{~h}$ at room temperature. Antigen-antibody reactions were detected using enhanced chemiluminescence (ECL Plus Western Blotting Detection System; GE Healthcare, UK).

\section{Results}

3.1. Interference of E. histolytica Gln6Pi Expression with siRNAs. Treatment of E. histolytica trophozoites with the two siRNA probes, individually or mixed, at amounts between 5 and $40 \mu \mathrm{g}$ per $5 \times 10^{5}$ cells did not affect the viability and replication of the parasites (data not shown). As expected, the transfected trophozoites showed decreased expression of Gln6PI RNA in RT-PCR assays when the parasites were treated with either siRNA (data not shown). However, the interference effect was greater when the parasite was treated with the mix of siRNAs. The effect was dose dependent with the mix of $5 \mu \mathrm{g}$ of each siRNA inhibiting about $24 \%$ and the mix of $40 \mu \mathrm{g}$ inhibiting almost $85 \%$ of Gln6Pi expression, when compared to the basal expression of Gln6Pi in untreated trophozoites and trophozoites transfected with the scrambled sequence, where the basal expression of Gln6Pi was similar (Figure 1).

Interference with expression of Gln6Pi was also demonstrated by western blot analysis of protein levels in extracts from interfered trophozoites. As shown in Figure 2, expression of a $37 \mathrm{kDa}$ band corresponding to the molecular weight expected for Gln6Pi decreased depending on the dose of siRNA used. Thus, a mix of $5 \mu \mathrm{g}$ of each siRNA inhibited Gln6Pi expression in about $47 \%$, whereas a mix of $40 \mu \mathrm{g}$ of each siRNA blocked completely the expression of Gln6Pi when compared with the basal protein expression of the enzyme. No effect on the expression of Gln6Pi protein was observed with the scrambled sequence (Figure 2).

3.2. Effect of Gln6Pi Knockdown on the Induction of CLS. Once confirmed the downregulation of Gln6Pi expression in the interfered trophozoites, the parasites were subjected to encystment induction by treatment with hydrogen peroxide plus metal dications as indicated. Addition of $4 \mathrm{mM} \mathrm{H}_{2} \mathrm{O}_{2}$ plus dications for $6 \mathrm{~h}$ to parasite cultures at $37^{\circ} \mathrm{C}$ induced differentiation of trophozoites to CLS at different rates depending on the concentration of siRNA mix used for interference (Table 2). Thus, the conversion rate (defined as the percentage of cells resistant to $0.5 \%$ sarkosyl during $10 \mathrm{~min}$ ) decreased as siRNA concentrations increased, ranging from $27 \%$ with $5 \mu \mathrm{g}$ to $5 \%$ with $40 \mu \mathrm{g}$ of each siRNA per culture. Accordingly, 


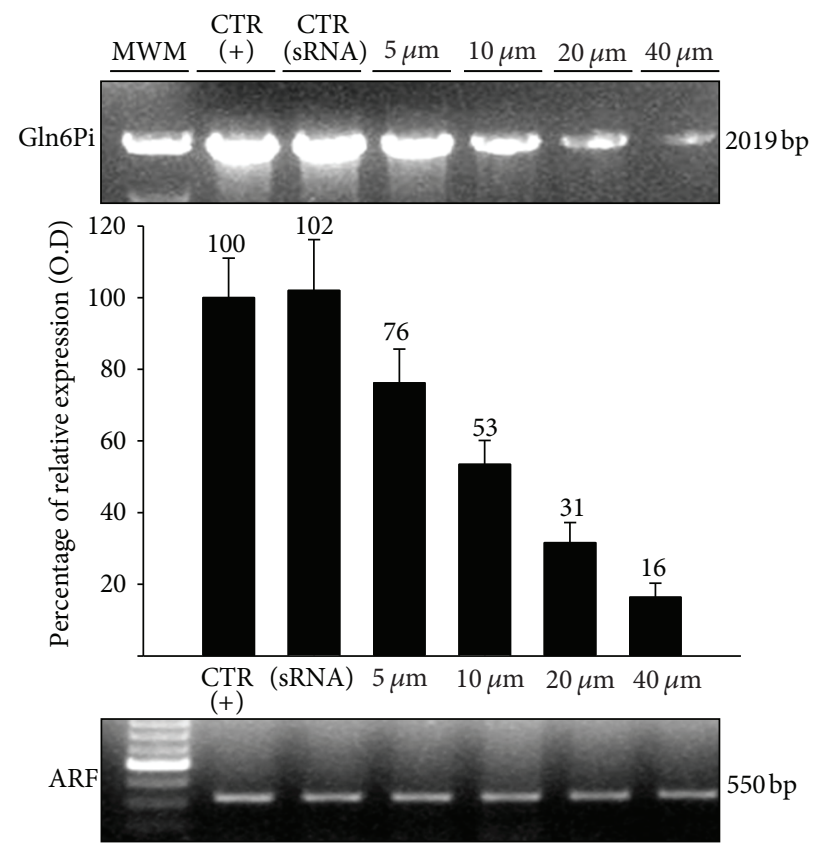

FIGURE 1: Relative expression of E. histolytica mRNA in trophozoites transfected with different amounts of a mix of si 154-Gln6Pi and si 229 -Gln6Pi determined by RT-PCR. Trophozoites (about $5 \times 10^{5}$ cells) were transfected with the siRNAs mix at indicated amounts by soaking during $16 \mathrm{~h}$ at $37^{\circ} \mathrm{C}$. Afterwards, mRNA was extracted and RT-PCR performed using oligonucleotides showed in Table 1. Relative expression was determined by densitometry with respect to the constitutive expression of E. histolytica ADP-ribosylating factor (ARF), which was also used as loading control. CTR (+): basal expression of Gln6Pi in trophozoites; CTR (siRNA): trophozoites transfected with scrambled-sequence siRNA-A.

viability of parasites determined by FDA staining decreased after sarkosyl treatment from $69 \%$ with $5 \mu \mathrm{g}$ to $12 \%$ with $40 \mu \mathrm{g}$ of each siRNA. As shown in Table 2, viability of trophozoites previous to the sarkosyl treatment was not critically affected by the transfection, ranging between 87 , and $95 \%$, suggesting that the transfection procedure is not toxic to the parasite.

\subsection{Effect of Gln6Pi Knockdown on Chitin Expression. In} agreement with the reduction of cell conversion rates, the increasing concentration of siRNA mix reduced the percentage of cells positive for calcofluor white staining, a disodium salt that detects specifically polysaccharides with $\beta$ 1-3 and $\beta$ 1-4 linkages present in cellulose and chitin (Table 2 and Figure 3). As mentioned before, transfection of $E$. histolytica trophozoites with siRNAs did not affect viability, independently of the concentration of siRNA used (Figures 3(a), 3(d), and 3(g)).

Parasites untransfected or transfected with the single si 154-Gln6Pi at $40 \mu \mathrm{g}$ showed identical percentage of chitin positive cells (conversion rate of around 30\%) after CLS induction followed by sarkosyl treatment (Figures 3(b) and $3(\mathrm{e})$ ). This result is in agreement with the scarce effect of a single siRNA on Gln6Pi expression as mentioned above.

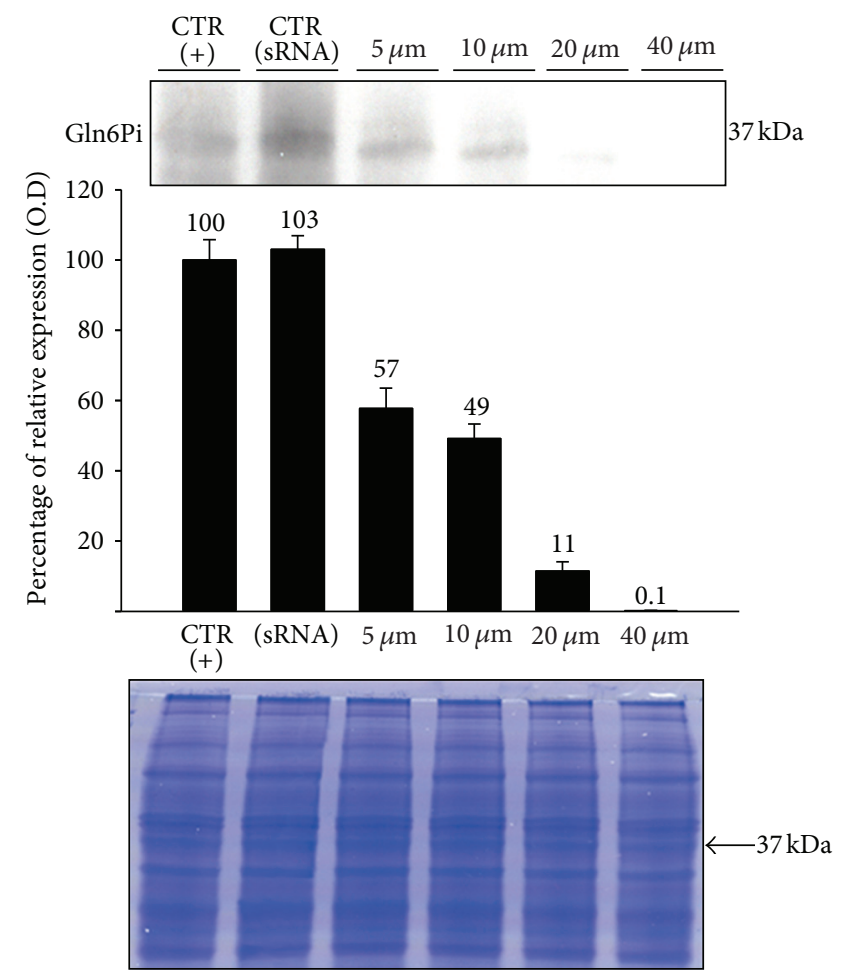

Figure 2: Levels of E. histolytica Gln6Pi protein expression in trophozoites transfected with different amounts of a mix of si 154Gln6Pi and si 229-Gln6Pi determined by western blot. Trophozoites (about $5 \times 10^{5}$ cells) were tranfected with siRNAs mix at indicated amounts by soaking during $16 \mathrm{~h}$ at $37^{\circ} \mathrm{C}$. Afterwards, total extracts were prepared in the presence of protease inhibitors, run in SDSPAGE, and transferred to nitrocellulose paper. Gln6Pi $(37 \mathrm{kDa})$ was identified by using a mouse anti-human Gln6Pi polyclonal antibody and revealed using ECL. Relative expression was determined by densitometry taking basal expression of Gln6Pi in untreated trophozoites as 100 percent (CTR (+)). CTR (siRNA): trophozoites transfected with scrambled-sequence siRNA-A. The amount of protein loaded in each lane is shown in a Coomassie stained gel.

In contrast, trophozoites transfected with the mix of $40 \mu \mathrm{g}$ of each siRNA showed a marked reduction in the number of chitin positive cells (Figure 3(h)), in agreement with the knockdown of Gln6Pi expression. In addition, the treated cells did not show morphological and structural features of encystment, such as refringence and multinucleation (data not shown). Noteworthy, most of the chitin positive cells were viable as determined by FDA staining (Figures 3(c), 3(f), and 3(i)). Data on viability after CLS induction, conversion rates, and calcofluor positive CLS in trophozoites transfected with different concentrations of siRNA mix are resumed in Table 2. In general, transfection with the different concentration of siRNA mix had a dose-dependent direct relationship effect on al parameters.

\section{Discussion}

Encystation is a pivotal process in the life cycle of $E$. histolytica and is indispensable for its transmission. However, 

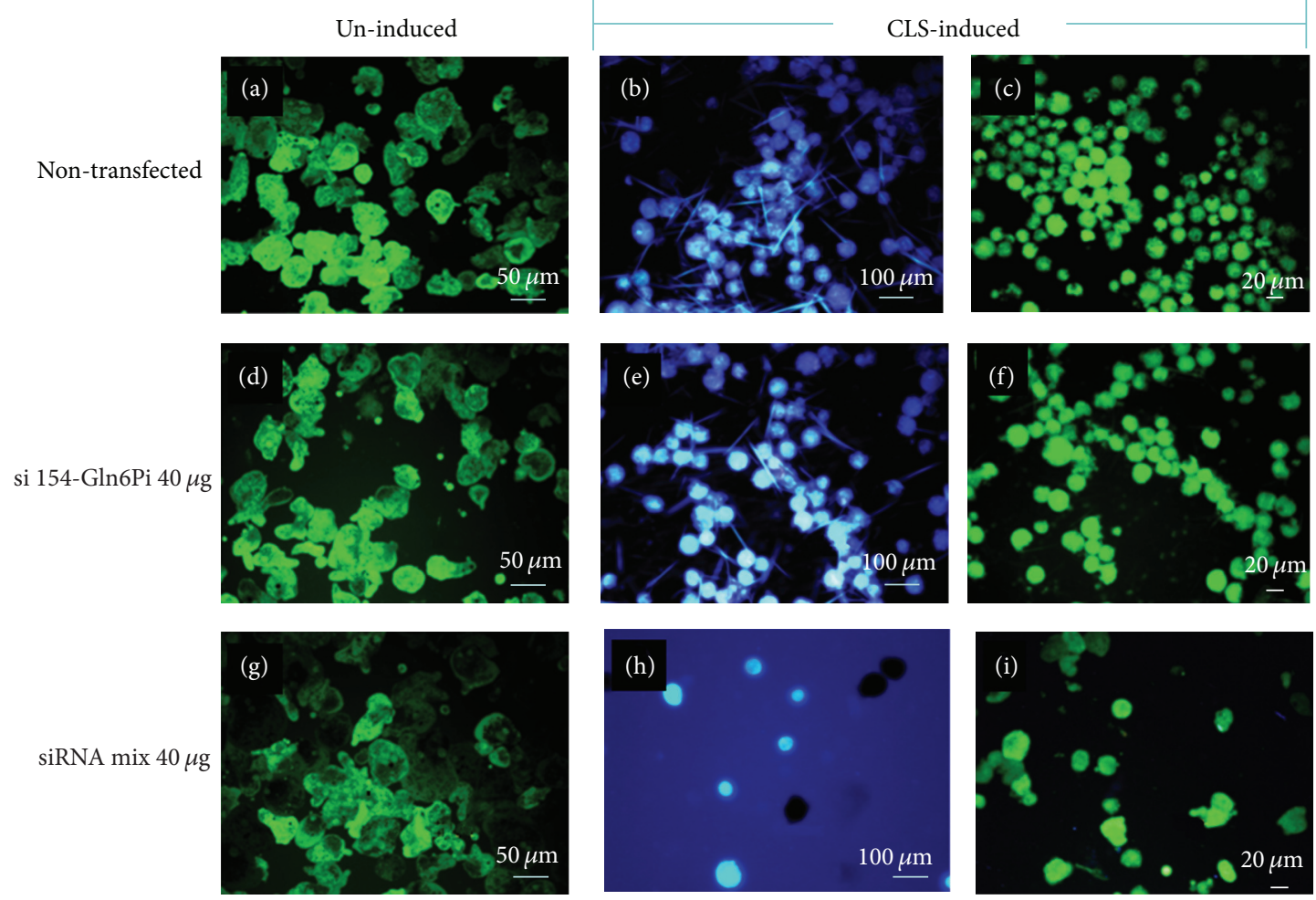

FIGURE 3: Expression of chitin and viability of Gln6Pi silenced trophozoites after CLS induction and detergent treatment. Untransfected trophozoites or transfected with $40 \mu \mathrm{g}$ of si $154-\mathrm{Gln} 6 \mathrm{Pi}$ or $40 \mu \mathrm{g}$ of each si 154-Gln6Pi and si 229-Gln6Pi (siRNA mix $40 \mathrm{ug}$ ) were induced to CLS followed by treatment with $0.5 \%$ sarkosyl. Cells were stained with FDA for viability before ((a), (d), and (g)) and after ((c), (f), and (i)) CLS induction and detergent treatment. Calcofluor white staining is shown for cells after CLS induction and detergent treatment ((b), (e), and (h)). Viable cells are observed in green and chitin-positive cells are whitish blue under UV light microscopy.

TABLE 2: Effect of Gln6Pi knockdown by a mix of si 154-Gln6Pi and si 229-Gln6Pi siRNAs on viability, conversion rate and chitin expression of trophozoites induced to CLS.

\begin{tabular}{|c|c|c|c|c|c|}
\hline (siRNA mix) & $\begin{array}{c}\text { Interference } \\
\text { trophozoites } \\
\text { viability (FDA) } \\
(\% \pm \text { S.D. })\end{array}$ & $\begin{array}{c}\text { Treatment } \\
\text { solution }{ }^{\mathrm{a}} / \text { incubation } \\
\text { time }\end{array}$ & $\begin{array}{l}\text { Conversion rate } \\
(\% \pm \text { S.D. })^{\mathrm{b}}\end{array}$ & $\begin{array}{c}\text { Viability } \\
\text { (FDA) } \\
(\% \pm \text { S.D. })\end{array}$ & $\begin{array}{c}\text { Staining calcofluor } \\
\text { white } \\
(\% \pm \text { S.D. })\end{array}$ \\
\hline $5 \mu \mathrm{g}$ & $95 \pm 3.6$ & $4 \mathrm{mM} / 6 \mathrm{~h}$ & $27 \pm 4.4$ & $69 \pm 5$ & $95 \pm 2$ \\
\hline $10 \mu \mathrm{g}$ & $91 \pm 1.5$ & $4 \mathrm{mM} / 6 \mathrm{~h}$ & $19 \pm 5.3$ & $65 \pm 4.5$ & $90 \pm 8$ \\
\hline $20 \mu \mathrm{g}$ & $90 \pm 2.3$ & $4 \mathrm{mM} / 6 \mathrm{~h}$ & $9 \pm 3$ & $36 \pm 1.1$ & $65 \pm 2$ \\
\hline $40 \mu \mathrm{g}$ & $87 \pm 3$ & $4 \mathrm{mM} / 6 \mathrm{~h}$ & $5 \pm 2.1$ & $12 \pm 2.8$ & $15 \pm 4.8$ \\
\hline
\end{tabular}

${ }^{\mathrm{a}}$ Hydrogen peroxide $30 \%$ containing traces of several dications, see Section 2.2 .

${ }^{\mathrm{b}}$ Three independent experiments were done by triplicate.

Conversion rate: percentage of cells that were resistant to $0.5 \%$ sarkosyl.

FDA: fluorescein diacetate.

S.D.: standard deviation.

the luminal stimuli triggering the process and the molecular mechanisms responsible for the stage conversion are still unknown. Recently, we have reported a reproducible in vitro treatment to induce cyst-like structures with most of the features of a mature cyst, including multinucleation and a chitin wall, by using a combination of hydrogen peroxide and metallic dications [3]. In the same report, we also gave evidence supporting the association between the overexpression of glucosamine-6-phosphate isomerase (Gln6Pi), the first enzyme in the theoretical route of chitin biosynthesis in amoeba, and the onset of the encystment process assayed by RT-PCR.

In the present work, we analyzed the effect of silencing the expression of Gln6Pi gene on encystment of E. histolytica trophozoites using synthetic siRNA duplexes and the soaking method, as previously described for gene silencing in this parasite $[22,23]$. As expected, transfection of $E$. histolytica trophozoites with a mixture of two siRNA duplexes 
during $15 \mathrm{~h}$ resulted in the knockdown of Gln6Pi mRNA levels in a dose-dependent manner. The mRNA levels were decreased by $85 \%$ whereas the levels of protein expression were decreased by almost $100 \%$ when parasites were soaked in a mix of $40 \mu \mathrm{g}$ of each siRNA. Our results are in agreement with the results of knockdown of $\gamma$-tubulin RNA and protein levels in respect of siRNA concentrations used [22]. In contrast to Giardia lamblia, where two Gln6Pi genes were identified [24], in silico analysis carried out by our group using the E. histolytica genome database suggests that this parasite may have a single gene coding for a putative Gln6Pi (data not shown). This could explain why a single interference with a mixture of two siRNAs was able to completely block the expression of the enzyme when assayed by WB. However, the exact molecular mechanism of silencing in amoeba is still unknown and waiting for demonstration, though the iRNA machinery and small RNAs are evidently present in E. histolytica (reviewed in $[17,18]$ ). In this regard, several studies on E. histolytica G3 strain, an in vitro obtained strain stably silenced for the amoebapore A gene, have suggested that transcriptional and posttranscriptional mechanisms could be involved in amoeba iRNA silencing [25-28].

The levels of downregulation of Gln6Pi expression correlated with the decrease in the rates of E. histolytica encystment under induction treatment, as judged by increased susceptibility to detergent and a proportional decrease in the production of chitin-positive structures and multinucleation. Therefore, the inhibition of the Gln6Pi expression resulted in the inhibition of chitin cyst wall synthesis making the cells induced for encystment susceptible to the detergent treatment quite similar to the uninduced trophozoites. This result is in agreement with our previous proposal of Gln6Pi being the first enzyme of the pathway leading to the synthesis of $\beta$ - $(1,4)$-linked $\mathrm{N}$-acetylglucosamine homopolymer, the main constituent of chitin in E. histolytica, which in combination with other proteins such as Jessie, Jacob, and the Gal-binding lectin give place to a surface hard cover that confers resistance to harmful environmental agents, facilitating the parasite's survival and dissemination (reviewed in [8]). Thus, in combination with our previous observations [3], the present results suggest that E. histolytica possesses a functional metabolic pathway of chitin synthesis similar to Giardia, where the isomerization of fructose-6-phosphate to glucosamine-6-phosphate by Gln6Pi seems to be critical. However, the activities of the other four theoretical enzymes of the pathway [29] were not conducted in this study and it is a matter of further studies in our group.

The results reported here also support the efficiency of hydrogen peroxide plus dications treatment in in vitro triggering the encystment process in E. histolytica trophozoites. The fact that stage conversion induced by hydrogen peroxide plus dications is inhibited by Gln6Pi interference, suggests that oxidative stress is able to activate, directly or indirectly, the expression of the Gln6Pi gene. In this regard, we have evidence of hydrogen peroxide response elements present in the promoter of Gln6Pi gene (data not published) that could be involved in its over-expression, in agreement with evidence of multiple genes responsive to oxidative stress having a role in cellular differentiation and many other physiological functions in eukaryotes (reviewed in [4]). However, studies on the infectivity of CLS are necessary in order to demonstrate that they are really mature cysts, and therefore, that interference of Gln6Pi will in fact inhibit the encystment in vivo. As mentioned above, studies on the role of the other four enzymes from the theorical pathway of chitin biosynthesis are also necessary and are actually being carried out in our laboratory.

In general, our results suggest that the encystment of $E$. histolytica is dependent on the upregulation of the Gln6Pi enzyme, which controls the onset of chitin synthesis under oxidative stress conditions.

\section{Acknowledgments}

This work was supported by CONACyT Grant 167788 (J. C. Carrero) and DGAPA Grant IN213611 (J. C. Carrero). J. H. Aguilar-Díaz wants to thank CONACyT for a fellowship to obtain the P.h.D in the postgraduate program of Biological Sciences at UNAM. The authors also thank Pavel Petrosyan for assistance in siRNA assays and Mario Néquiz for amoeba culturing.

\section{References}

[1] WHO, "Geneva, Switzerland. Weekly Epidemiological Record," Amoebiasis from, vol. 72, pp. 97-100, 4 April 1997.

[2] E. W. Mc Connachie, "Studies on Entamoeba invadens Rodhain, 1934, in vitro and its relationship to some other species of Entamoeba," Parasitology, vol. 45, no. 3-4, pp. 452-481, 1955.

[3] H. Aguilar-Díaz, M. Díaz-Gallardo, J. P. Laclette, and J. C. Carrero, "In vitro induction of Entamoeba histolytica cyst-like structures from trophozoites," PLoS Neglected Tropical Diseases, vol. 4, no. 2, article e607, 2010.

[4] J. Aguirre, M. Ríos-Momberg, D. Hewitt, and W. Hansberg, "Reactive oxygen species and development in microbial eukaryotes," Trends in Microbiology, vol. 13, no. 3, pp. 111-118, 2005.

[5] D. Eichinger, "Encystation of entamoeba parasites," BioEssays, vol. 19, no. 7, pp. 633-639, 1997.

[6] D. Eichinger, "Encystation in parasitic protozoa," Current Opinion in Microbiology, vol. 4, no. 4, pp. 421-426, 2001.

[7] S. Said-Fernández, E. Campos-Góngora, F. González-Salazar, H. G. Martínez-Rodríguez, J. Vargas-Villarreal, and J. M. Viader-Salvadó, "Mg2+, Mn2+, and Co2+ stimulate Entamoeba histolytica to produce chitin-like material," The Journal of Parasitology, vol. 87, pp. 919-923, 2001.

[8] H. Aguilar-Díaz, J. C. Carrero, R. Argüello-García, J. P. Laclette, and J. Morales-Montor, "Cyst and encystment in protozoan parasites: optimal targets for new life-cycle interrupting strategies?” Trends in Parasitology, vol. 27, pp. 450-458, 2011.

[9] E. L. Jarroll, P. T. Macechko, P. A. Steimle et al., "Regulation of carbohydrate metabolism during giardia encystment," Journal of Eukaryotic Microbiology, vol. 48, no. 1, pp. 22-26, 2001.

[10] A. B. Lopez, M. T. Hossain, and H. van Keulen, "Giardia intestinalis glucosamine 6-phosphate isomerase: the key enzyme to encystment appears to be controlled by ubiquitin attachment," Journal of Eukaryotic Microbiology, vol. 49, no. 2, pp. 134-136, 2002. 
[11] A. Fire, S. Xu, M. K. Montgomery, S. A. Kostas, S. E. Driver, and C. C. Mello, "Potent and specific genetic interference by doublestranded RNA in caenorhabditis elegans," Nature, vol. 391, no. 6669, pp. 806-811, 1998.

[12] C. D. Novina and P. A. Sharp, “The RNAi revolution," Nature, vol. 430, no. 6996, pp. 161-164, 2004.

[13] R. H. A. Plasterk, "RNA silencing: the Genome's immune system," Science, vol. 296, no. 5571, pp. 1263-1265, 2002.

[14] P. D. Zamore, T. Tuschl, P. A. Sharp, and D. P. Bartel, "RNAi: double-stranded RNA directs the ATP-dependent cleavage of mRNA at 21 to 23 nucleotide intervals," Cell, vol. 101, no. 1, pp. 25-33, 2000.

[15] E. Bernstein, A. A. Caudy, S. M. Hammond, and G. J. Hannon, "Role for a bidentate ribonuclease in the initiation step of RNA interference," Nature, vol. 409, no. 6818, pp. 363-366, 2001.

[16] S. M. Elbashir, W. Lendeckel, and T. Tuschl, "RNA interference is mediated by 21 - and 22-nucleotide RNAs," Genes and Development, vol. 15, no. 2, pp. 188-200, 2001.

[17] C. F. Solis and N. Guillén, "Silencing genes by RNA interference in the protozoan parasite Entamoeba histolytica," Methods in Molecular Biology, vol. 442, pp. 113-128, 2008.

[18] H. Zhang, J. M. Pompey, and U. Singh, "RNA interference in Entamoeba histolytica: implications for parasite biology and gene silencing," Future Microbiology, vol. 6, no. 1, pp. 103-117, 2011.

[19] L. S. Diamond, D. R. Harlow, and C. C. Cunnick, "A new medium for the axenic cultivation of Entamoeba histolytica and other Entamoeba," Transactions of the Royal Society of Tropical Medicine and Hygiene, vol. 72, no. 4, pp. 431-432, 1978.

[20] D. G. Schupp, M. M. Januschka, L. A. F. Sherlock et al., "Production of viable Giardia cysts in vitro: determination by fluorogenic dye staining, excystation, and animal infectivity in the mouse and mongolian gerbil," Gastroenterology, vol. 95, no. 1, pp. 1-10, 1988.

[21] S. K. Ghosh, J. Field, M. Frisardi et al., "Chitinase secretion by encysting Entamoeba invadens and transfected Entamoeba histolytica trophozoites: localization of secretory vesicles, endoplasmic reticulum, and Golgi apparatus," Infection and Immunity, vol. 67, no. 6, pp. 3073-3081, 1999.

[22] L. Vayssié, M. Vargas, C. Weber, and N. Guillén, "Doublestranded RNA mediates homology-dependant gene silencing of $\gamma$-tubulin in the human parasite Entamoeba histolytica," Molecular and Biochemical Parasitology, vol. 138, no. 1, pp. 21-28, 2004.

[23] G. Kaur and A. Lohia, "Inhibition of gene expression with double strand RNA interference in Entamoeba histolytica," Biochemical and Biophysical Research Communications, vol. 320, no. 4, pp. 1118-1122, 2004.

[24] H. Van Keulen, P. A. Steimle, D. A. Bulik, R. K. Borowiak, and E. L. Jarroll, "Cloning of two putative Giardia lamblia glucosamine 6-phosphate isomerase genes only one of which is transcriptionally activated during encystment," Journal of Eukaryotic Microbiology, vol. 45, no. 6, pp. 637-642, 1998.

[25] R. Bracha, Y. Nuchamowitz, and D. Mirelman, "Transcriptional silencing of an amoebapore gene in Entamoeba histolytica: molecular analysis and effect on pathogenicity," Eukaryotic Cell, vol. 2, no. 2, pp. 295-305, 2003.

[26] M. Anbar, R. Bracha, Y. Nuchamowitz, Y. Li, A. Florentin, and D. Mirelman, "Involvement of a short interspersed element in epigenetic transcriptional silencing of the amoebapore gene in Entamoeba histolytica," Eukaryotic Cell, vol. 4, no. 11, pp. 1775-1784, 2005.
[27] R. C. MacFarlane and U. Singh, "Loss of dsRNA-based gene silencing in Entamoeba histolytica: implications for approaches to genetic analysis," Experimental Parasitology, vol. 119, no. 2, pp. 296-300, 2008.

[28] H. Irmer, I. Hennings, I. Bruchhaus, and E. Tannich, "tRNA gene sequences are required for transcriptional silencing in Entamoeba histolytica," Eukaryotic Cell, vol. 9, no. 2, pp. 306-314, 2010.

[29] B. Loftus, I. Anderson, R. Davies et al., "The genome of the protist parasite Entamoeba histolytica," Nature, vol. 433, no. 7028, pp. 865-868, 2005. 

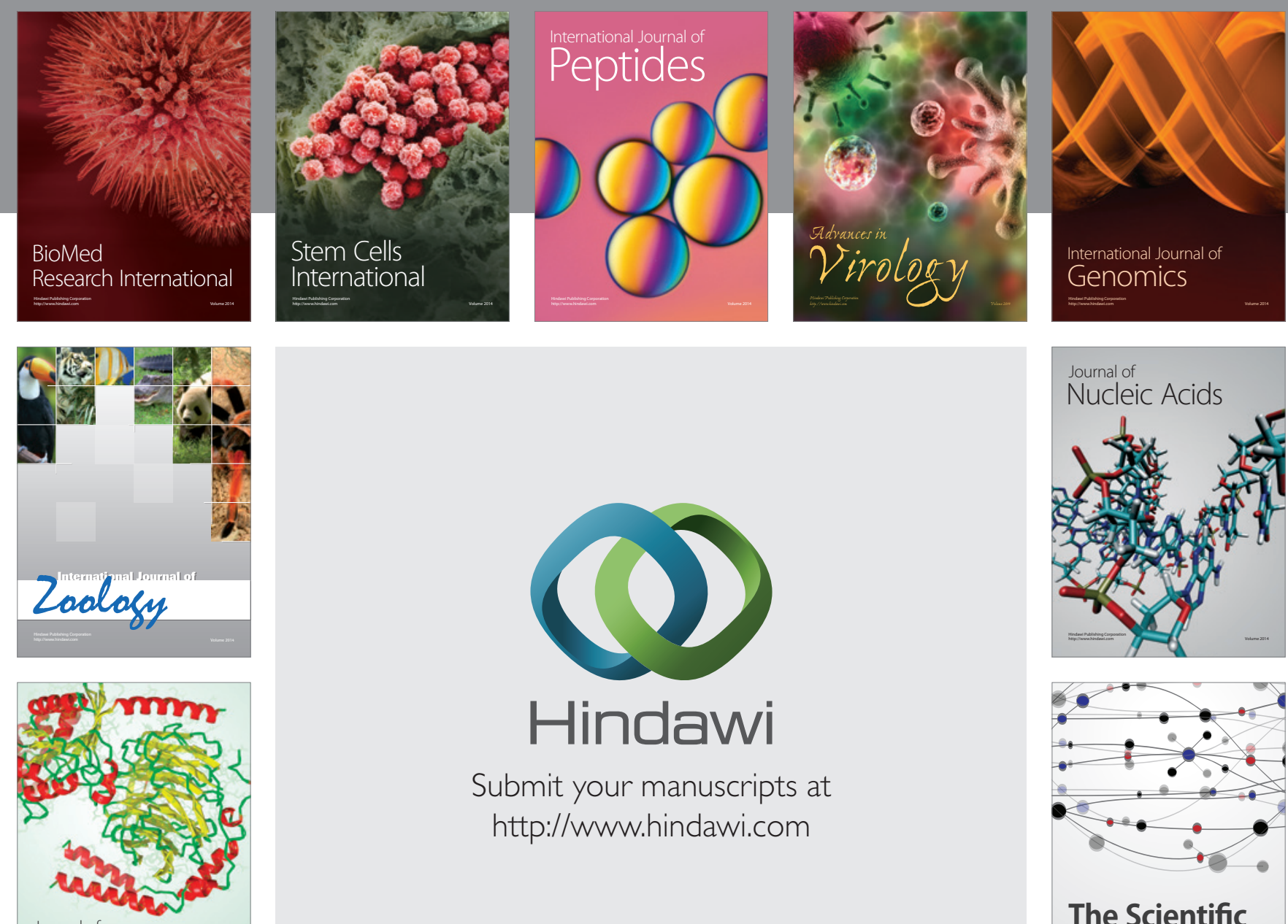

Submit your manuscripts at

http://www.hindawi.com

Journal of
Signal Transduction
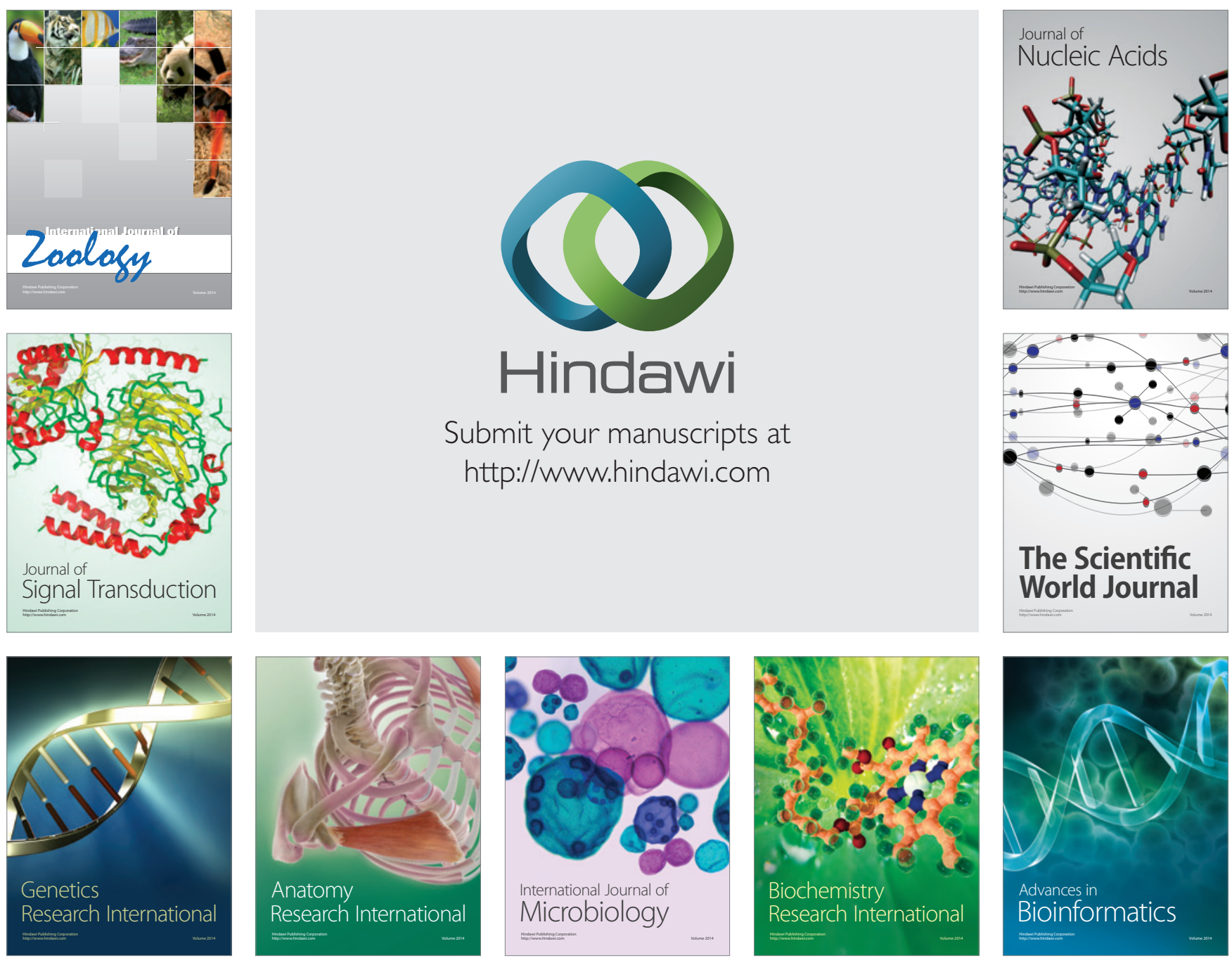

The Scientific World Journal
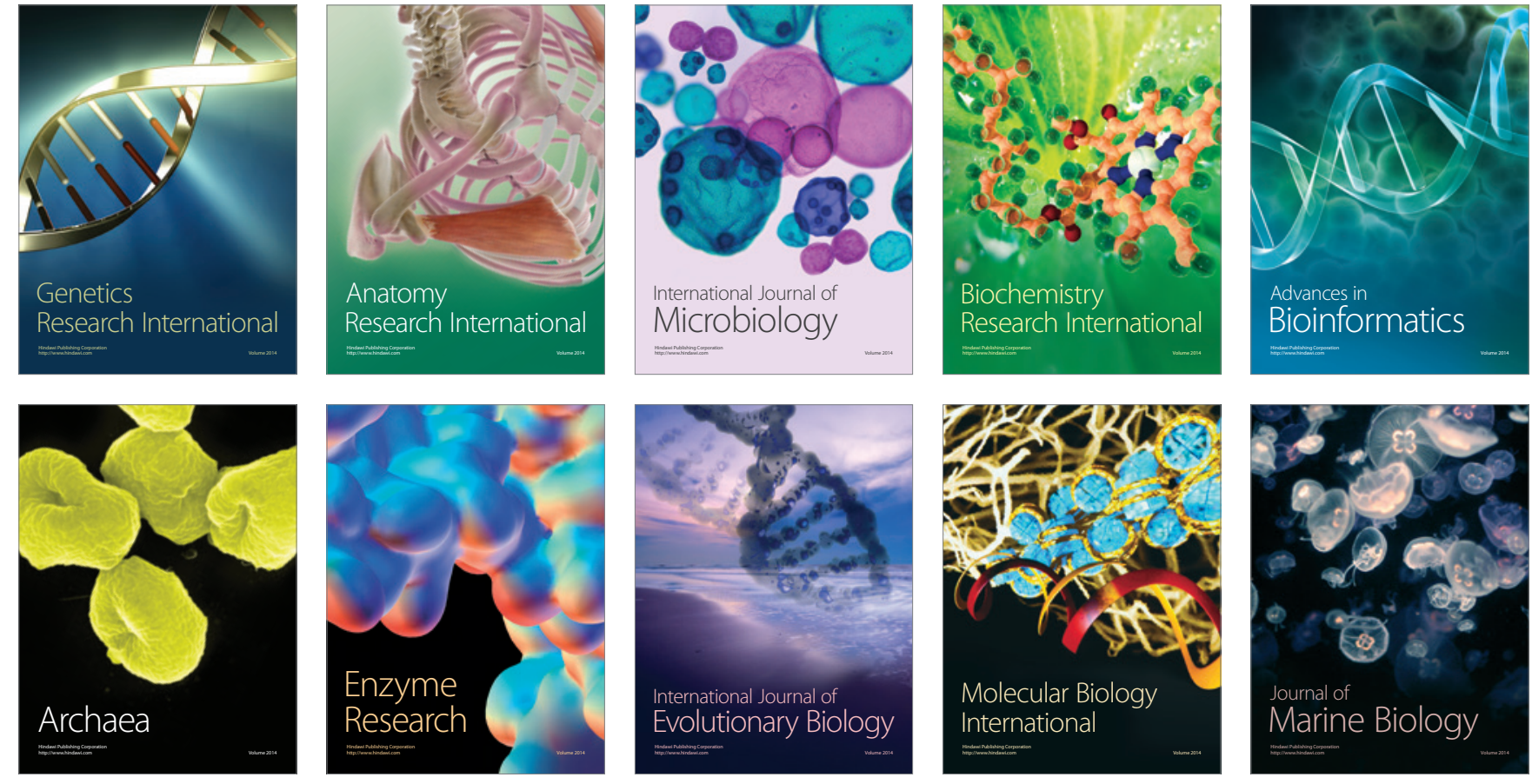